Karl-Franzens Universität Graz

Technische Universität Graz

Medizinische Universität Graz

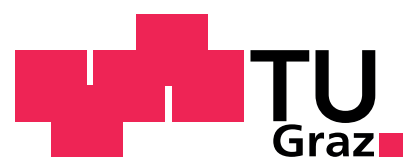

\title{
Optimal control for elliptic systems with pointwise euclidean norm constraints on the controls
}

\author{
Xiliang Lu Karl Kunisch
}


SFB sponsors:

- Austrian Science Fund (FWF)

- University of Graz

- Graz University of Technology

- Medical University of Graz

- Government of Styria

- City of Graz

Das Land

Steiermark

Stadt $\mathbf{G} \mathbf{R}$ A Z $\mathbf{Z}$ Wissenschaft 


\title{
OPTIMAL CONTROL FOR ELLIPTIC SYSTEMS WITH POINTWISE EUCLIDEAN NORM CONSTRAINTS ON THE CONTROLS
}

\author{
KARL KUNISCH AND XILIANG LU
}

\begin{abstract}
Optimal control for an elliptic system with pointwise Euclidean norm constraints on the control variables is investigated. First order optimality conditions are derived in a manner that is amenable for numerical realisation. An efficient semismooth Newton algorithm is proposed based on this optimality system. Numerical examples are given to validate the superlinear convergence of the semismooth Newton algorithm.
\end{abstract}

AMS Subject 49M15, 65J15

\section{INTRODUCTION}

Optimal control problems with control constraints and their numerical realization have been studied intensively in recent papers, see e.g. [4, 5, 6, 7, 12, 15, 18] and the references cited there. In most previous discussions, the control constraints are taken as simple constraints (e.g unilateral or bilateral constraints). For systems with vector-valued controls the constraints on the controls may have more complicated structure. In [19, 20, 21] first order necessary and second order sufficient optimality conditions for general convex constraints are obtained. In [20] a numerical procedure is proposed which, however, does not concretely exploit the structure of the constraint set and for which no convergence analysis is given. In [14] an abstract semismooth Newton approach is investigated that is applicable for constraints on vector-valued controls. The detailed realization in that paper is also carried out for pointwise constraints on the components of the control vector. In [11] and [9] semismooth Newton methods are investigated for affine constraints and for constraints with convex polygonal type.

In this paper we focus on the investigation of the semismooth Newton approach for pointwise Euclidean norm constraints (i.e., $\vec{u}(\mathbf{x}) \in K$, where $K$ is an ellipse) on the control variables. We provide a convergence analysis in function spaces and demonstrate that the numerical behavior is in accordance with the theory. In the context of partial differential equations, which we have in mind here, vector valued controls arise, in optimal control involving systems of equations as for instance in the context of the incompressible Stokes equations or the elasticity equations, where the control acts as external force which is bounded in its magnitude. Here we consider linear systems of equations. To treat nonlinear systems with the

\footnotetext{
Key words and phrases. optimal control, control constraints, semismooth Newton method, nonlinear constraint.

The work of K.K. was in part supported by Austrian Science Foundation under SFB, "Mathematical Optimization and Applications in Biomedical Sciences". The work of X. Lu was supported by National Natural Science Foundation of China (11101316).
} 
proposed techniques one can either apply a sequential quadratic programming procedure with an outer loop, within which the nonlinear differential equations are replaced by linearized ones, or one treats the nonlinear system simultaneously with the non-differentiable constraint. The analysis of this extension is conceptually quite straightforward and involves primarily regularity theory of partial differential equations.

Let us also recall that quadratic problems with Euclidean norm constraints on the optimization variables arise as auxiliary problems in trust-region methods, see e.g. [2] for the finite- and [17] for the infinite dimensional cases. The focus in this context lies in the succinct update of the trust region radius and efficient suboptimal solutions to the auxiliary problems.

For optimal control problem with unilateral or bilateral control constraints, semismooth Newton method have been proved to be an efficient, locally superlinearly convergent technique. In [5, 7] an algebraic manipulation linking a parameter of the complementarity system to the weight of the control cost, denoted by $\alpha$ below, was the key technical step for proving superlinear convergence of the semismooth Newton method. Here we cannot rely on this technique, but rather we have to express the complementarity system in a manner which lends itself to verify semismoothness in appropriately chosen function spaces.

Let $\Omega$ be an open bounded domain in $\mathbb{R}^{n}$, for $n=2$ or 3 with smooth boundary $\partial \Omega$. We choose a quadratic tracking type cost functional

$$
J(\vec{y}, \vec{u})=\frac{1}{2}\left\|\vec{y}-\vec{y}_{d}\right\|^{2}+\frac{\alpha}{2}\|E \vec{u}\|^{2},
$$

where $\alpha$ is a positive constant and the target function $\vec{y}_{d} \in\left(L^{2}(\Omega)\right)^{m}$. The control variable $\vec{u}$ has support in a sub-domain $\tilde{\Omega} \subset \Omega$, and the operator $E:\left(L^{2}(\tilde{\Omega})\right)^{m} \mapsto$ $\left(L^{2}(\Omega)\right)^{m}$ denotes the extension-by-zero operator. The state and control variables satisfy an elliptic system with zero Dirichlet boundary condition:

$$
\Lambda \vec{y}=E \vec{u},
$$

where the vectors $\vec{y}$ and $\vec{u}$ have $m$-components and the elliptic operator $\Lambda$ is a bounded linear operator $\left(H_{0}^{1}\right)^{m} \mapsto\left(H^{-1}\right)^{m}$. Moreover, we assume that for $v \in$ $\left(L^{2}(\Omega)\right)^{m}$ we have $\Lambda^{-1} v \in\left(H_{0}^{1}(\Omega)\right)^{m} \cap\left(H^{2}(\Omega)\right)^{m}$ and for a constant $C$, independent of $v$

$$
\left\|\Lambda^{-1} v\right\|_{2} \leq C\|v\|,
$$

where $\|\cdot\|_{2}$ denotes the norm on the vector - valued $H^{2}(\Omega)$ space. Further notation will be specified below. The optimal control problem is given by

\section{Problem 1.1.}

$\min J(\vec{y}, \vec{u})$, such that equations (1.1) hold and $|B \vec{u}(\mathbf{x})-\vec{b}| \leq 1$, a.e. in $\Omega$.

The $m \times m$ matrix $B$ is invertible, $\vec{b}$ is a given vector in $\mathbb{R}^{m}$, and $|\cdot|$ denotes the Euclidean norm in $\mathbb{R}^{m}$. This model problem is in the linear-quadratic form, but let us recall such problems also arise as the auxiliary problems in the sequentialquadratic programming approach to genuinely nonlinear optimal control problems.

The paper is organized as follows. The optimality system is derived in section 2. In section 3 , we focus on a special case, where $\tilde{\Omega}=\Omega, B=I$ and $\vec{b}=0$ to avoid some tedious notations. For this case, we give the details of super-linear 
convergence for a semismooth Newton algorithm. In section 4, we point out that our results can be generalized in several directions, i.e., for a general subdomain $\tilde{\Omega}$, a general invertible matrix $B$ and translation vector $\vec{b}$. In the last section 5 , numerical examples are given to depict the efficiency for the semismooth Newton Algorithm.

We use standard notation for the Sobolev spaces $W^{r, p}(\Omega)$ and $H^{r}(\Omega), r \in \mathbb{N}, 1 \leq$ $p \leq \infty$, and simplify the expression for the norm of elements in $H^{r}$ as $\|f\|_{r}=\|f\|_{H^{r}}$ and $\|f\|=\|f\|_{L^{2}}$. If the domain is not specified it is considered as $\Omega$. The vector function

$$
\vec{z} \in\left(W^{r, p}(\Omega)\right)^{m}=W^{r, p}\left(\Omega, \mathbb{R}^{m}\right)
$$

if and only if each coordinate of $\vec{z}$ is an element in $W^{r, p}(\Omega)$. We use $(\cdot, \cdot)$ as inner product in $L^{2}(\Omega)$ (for scalar functions) or $\left(L^{2}(\Omega)\right)^{m}$ (for vector functions). We recall that $H^{2}(\Omega)$ embeds continuously into $C(\Omega)$ since $n \leq 3$ by assumption.

\section{Optimality System}

We define the closed convex set $K_{m}$ in $\mathbb{R}^{m}$ by

$$
K_{m}=\left\{\vec{v} \in \mathbb{R}^{m},|B \vec{v}-\vec{b}| \leq 1\right\},
$$

and the subset $K$ in $\left(L^{2}(\tilde{\Omega})\right)^{m}$ by

$$
K=\left\{\vec{u} \in\left(L^{2}(\tilde{\Omega})\right)^{m}: \vec{u}(\mathbf{x}) \in K_{m} \text {, a.e. in } \tilde{\Omega}\right\} .
$$

It is clear that $K$ is a bounded convex closed cone in $\left(L^{2}(\tilde{\Omega})\right)^{m}$. By solving the governing equation, we find the control to state mapping $T:\left(L^{2}(\tilde{\Omega})\right)^{m} \mapsto\left(L^{2}\right)^{m}$, $\vec{y}=T \vec{u}$ has form $\vec{y}=\Lambda^{-1} E \vec{u}$. Hence the cost functional $J(\vec{y}, \vec{u})$ can equivalently be represented as

$$
\hat{J}(\vec{u})=J(T \vec{u}, \vec{u}),
$$

and Problem 1.1 can be rewritten in the following equivalent way:

$$
\min _{\vec{u} \in K} \hat{J}(\vec{u}) .
$$

Theorem 2.1. There exists a unique solution $\left(\vec{y}^{*}, \vec{u}^{*}\right)$ for Problem 1.1.

Proof. We consider its equivalent form (2.2). It can be checked that $\hat{J}$ is a lower semi-continuous convex functional in $\left(L^{2}(\tilde{\Omega})\right)^{m}$. Since $K$ is bounded, closed and convex subset of $\left(L^{2}(\tilde{\Omega})\right)^{m}$, standard arguments imply the existence of an optimal solution, and strict convexity implies its uniqueness.

Next we will establish the optimality system. Since the reduced cost functional $\vec{u} \mapsto \hat{J}(\hat{u})$ is quadratic and uniformly convex, the necessary and sufficient optimality condition can be written as

$$
\vec{u}^{*} \in K, \quad\left(\hat{J}^{\prime}\left(\vec{u}^{*}\right), \vec{u}-\vec{u}^{*}\right) \geq 0, \quad \forall \vec{u} \in K .
$$

It can be equivalently written as

$$
\vec{u}^{*}=P_{K}\left(\vec{u}^{*}-\theta \hat{J}^{\prime}\left(\vec{u}^{*}\right)\right)
$$


where $\theta$ is any positive constant and $P_{K}$ is the projection operator from $\left(L^{2}(\tilde{\Omega})\right)^{m}$ to $K$. By virtue of the definition of projection operator, we find that $(2.3)$ can be put into pointwise projection form (see also Lemma 2.2 in [9]):

$$
\vec{u}^{*}(\mathbf{x})=P\left(\vec{u}^{*}(\mathbf{x})-\theta \hat{J}^{\prime}\left(\vec{u}^{*}\right)(\mathbf{x})\right), \quad \text { a.e. in } \tilde{\Omega} .
$$

Here $P$ is the projection operator onto $K_{m}$ in $\mathbb{R}^{m}$. Since $\hat{J}\left(\vec{u}^{*}\right)=\frac{1}{2}\left\|T \vec{u}^{*}-\vec{y}_{d}\right\|^{2}+$ $\frac{\alpha}{2}\left\|E \vec{u}^{*}\right\|^{2}$, we have

$$
\hat{J}^{\prime}\left(\vec{u}^{*}\right)=T^{*}\left(T \vec{u}^{*}-\vec{y}_{d}\right)+\alpha E^{*} E \vec{u}^{*}=E^{*} \Lambda^{-*}\left(\vec{y}^{*}-\vec{y}_{d}\right)+\alpha \vec{u}^{*},
$$

where we use that $\vec{y}^{*}=T \vec{u}^{*}, E^{*}$ is a restriction operator from $\left(L^{2}(\Omega)\right)^{m}$ to $\left(L^{2}(\tilde{\Omega})\right)^{m}$ and hence $E^{*} E$ is identity operator on $\left(L^{2}(\tilde{\Omega})\right)^{m}$. Let $\vec{p}^{*}=-\Lambda^{-*}\left(\vec{y}^{*}-\vec{y}_{d}\right)$ and choose $\theta=\frac{1}{\alpha}$ in (2.4). Then

$$
\vec{u}^{*}(\mathbf{x})=P\left(\frac{1}{\alpha} E^{*} \vec{p}^{*}(\mathbf{x})\right), \quad \text { a.e. in } \tilde{\Omega} .
$$

It remains to characterize the projection $P$ in $\mathbb{R}^{m}$ explicitly. By definition, for any given vector $\vec{v} \in \mathbb{R}^{m}$, if $\vec{v} \in K_{m}$, then $P(\vec{v})=\vec{v}$. Otherwise, $|B P(\vec{v})-b|=1$ and the vector $\vec{v}-P(\vec{v})$ points to the outer normal direction of the tangent space to $K_{m}$ at $P(\vec{v})$, or equivalently, we can find a positive number $\eta=\eta(x)$, s.t.,

$$
\vec{v}-P(\vec{v})=\eta\left(B^{T}(B P(\vec{v})-\vec{b})\right) .
$$

Using the above explicit expression of $P$, equation (2.4) can be written equivalently in complementary form by introducing a Lagrange multiplier-like function $\lambda^{*}(\mathbf{x})$ :

$$
\begin{aligned}
& E^{*} \vec{p}^{*}(\mathbf{x})=\alpha \vec{u}^{*}(\mathbf{x})+\lambda^{*}(\mathbf{x}) B^{T}\left(B \vec{u}^{*}(\mathbf{x})-\vec{b}\right), \\
& \lambda^{*}(\mathbf{x}) \geq 0,\left|B \vec{u}^{*}(\mathbf{x})-\vec{b}\right| \leq 1, \lambda^{*}(\mathbf{x}) \cdot\left(\left|B \vec{u}^{*}(\mathbf{x})-\vec{b}\right|-1\right)=0 .
\end{aligned}
$$

This system holds a.e. in $\tilde{\Omega}$. Combing the definitions of the control to state mapping and of the dual variable $\vec{p}^{*}$, and the complementary equations (2.7) - (2.8), we have:

Theorem 2.2. The optimality system for Problem 1.1 is given by

$$
\text { Primal equation } \quad \Lambda \vec{y}^{*}=E \vec{u}^{*} \text {, }
$$

$$
\text { Adjoint equation } \quad \Lambda^{*} \vec{p}^{*}=\vec{y}_{d}-\vec{y}^{*},
$$

$$
\begin{aligned}
\text { Optimality condition } & E^{*} \vec{p}^{*}=\alpha \vec{u}^{*}+\lambda^{*} B^{T}\left(B \vec{u}^{*}-\vec{b}\right), \\
& \lambda^{*} \geq 0,\left|B \vec{u}^{*}-\vec{b}\right| \leq 1, \lambda^{*} \cdot\left(\left|B \vec{u}^{*}-\vec{b}\right|-1\right)=0,
\end{aligned}
$$

where the optimality condition is understood in the a.e. pointwise sense, and $\lambda^{*} \in$ $L^{\infty}(\tilde{\Omega})$. Moreover, the solution of the optimality system is unique.

Proof. The optimality system have already been shown in previous discussion. To argue that $\lambda^{*} \in L^{\infty}(\tilde{\Omega})$ we first note that $\lambda^{*}=0$ on the complement of the active set

$$
\mathcal{A}=\left\{\mathbf{x} \in \tilde{\Omega}:\left|B \vec{u}^{*}(\mathbf{x})-\vec{b}\right|=1\right\} .
$$

Moreover from the third equation in (2.9) we deduce that

(2.11) $\left(E^{*} \vec{p}^{*}(\mathbf{x})\right)^{T}\left(\vec{u}^{*}(\mathbf{x})-B^{-1} \vec{b}\right)=\alpha \vec{u}^{*}(\mathbf{x})^{T}\left(\vec{u}^{*}(\mathbf{x})-B^{-1} b\right)+\lambda^{*}(\mathbf{x})$ for $\mathbf{x} \in \mathcal{A}$. Since $\vec{u}^{*} \in\left(L^{\infty}(\tilde{\Omega})\right)^{m}$ and $\vec{p}^{*} \in\left(H^{2}(\Omega)\right)^{m} \hookrightarrow\left(L^{\infty}\right)^{m}$ for $n \leq 3$, we have $\lambda^{*} \in$ $L^{\infty}(\mathcal{A})$ and hence $\lambda^{*} \in L^{\infty}(\tilde{\Omega})$. 
Due to the strict convexity of this optimization problem, the first order optimality system is also sufficient. Then by the uniqueness of optimal pair $\left(\vec{u}^{*}, \vec{y}^{*}\right)$, we find the optimal adjoint state $\vec{p}^{*}$ is also unique. Moreover, the above argument shows the Lagrange multiplier $\lambda^{*}$ has an explicit form:

$$
\lambda^{*}(\mathbf{x})= \begin{cases}0 & \mathbf{x} \in \tilde{\Omega} \backslash \mathcal{A} \\ \left(E^{*} \vec{p}^{*}(\mathbf{x})-\alpha \vec{u}^{*}(\mathbf{x})\right)^{T}\left(\vec{u}^{*}(\mathbf{x})-B^{-1} b\right) & \mathbf{x} \in \mathcal{A} .\end{cases}
$$

Therefore the solution to the Lagrange multiplier is also unique.

Remark 2.3. The projection representation for the optimality condition was studied in earlier papers, see e.g. [19, 21]. Theorem 2.2 expresses the fact that the optimality condition in our Euclidean norm constraint case can be reformulated as a complementary condition. Later discussion will show that this optimality condition is equivalent to a nonlinear equation.

From now to the end of section 3 , we will focus on a special case, i.e., $\Omega=\tilde{\Omega}$, $B=I$ and $\vec{b}=0$. Extensions will be considered in Section 4. In this case, one can observe for any nonzero vector $\vec{v}$ in $\mathbb{R}^{m}$, its projection $P(\vec{v})$ is collinear to itself. Therefore, we have

$$
P(\vec{v})=\frac{\vec{v}}{\max (1,|\vec{v}|)} .
$$

The optimality condition for the special case reads

$$
\vec{u}^{*}(\mathbf{x})=P\left(\frac{1}{\alpha} \vec{p}^{*}(\mathbf{x})\right)=\frac{\vec{p}(\mathbf{x})}{\max \left(\alpha,\left|\vec{p}^{*}(\mathbf{x})\right|\right)} .
$$

Setting $\beta^{*}(\mathbf{x})=\max \left(\alpha,\left|\vec{p}^{*}(\mathbf{x})\right|\right)$, the complementary condition in (2.9) is replaced by a nonlinear equation and the whole optimality system is given by:

$$
\left\{\begin{array}{l}
\Lambda \vec{y}^{*}=\vec{u}^{*}, \\
\Lambda^{*} \vec{p}^{*}=\vec{y}_{d}-\vec{y}^{*}, \\
\vec{p}^{*}=\beta^{*} \vec{u}^{*}, \quad \beta^{*}=\max \left(\alpha,\left|\vec{p}^{*}\right|\right) .
\end{array}\right.
$$

From this optimality system, we deduce that the optimal solution $\left(\vec{y}^{*}, \vec{u}^{*}\right)$ enjoys additional regularity. This is important to prove superlinear convergence of the semismooth Newton algorithm in the next section.

Corollary 2.4. Given $\vec{y}_{d} \in\left(L^{2}\right)^{m}$, the optimal solution satisfies $\vec{u}^{*} \in\left(W^{1, p}\right)^{m}$, where $p=6$ for $n=3$, and $p<\infty$ for $n=2$ with $\Omega \subset \mathbb{R}^{n}$.

Proof. Let the index $p=6$ for $n=3$ and $p<\infty$ for $n=2$. From the second equation in (2.9) and since $\vec{y}, \vec{y}_{d} \in\left(L^{2}\right)^{m}$, and by the Sobolev embedding theorem (c.f. Theorem 4.31 in [1]), we have

$$
\vec{p}^{*} \in\left(H^{2}(\Omega)\right)^{m} \hookrightarrow\left(W^{1, p}(\Omega)\right)^{m} \hookrightarrow(C(\Omega))^{m} .
$$

This implies that $\left|\vec{p}^{*}\right| \in C(\Omega)$ and $\beta^{*}=\max \left(\alpha,\left|\vec{p}^{*}\right|\right) \in C(\Omega)$. Therefore $\vec{u}^{*}=\frac{\vec{p}^{*}}{\beta^{*}} \in$ $C(\Omega)$ as well. To check that the derivative is in $L^{p}$, we define two subsets of $\Omega$ by

$$
\Omega_{1}=\left\{\mathbf{x} \in \Omega:\left|\vec{p}^{*}(\mathbf{x})\right|>\frac{\alpha}{2}\right\}, \quad \Omega_{2}=\{\mathbf{x} \in \Omega:|\vec{p}(\mathbf{x})|<\alpha\} .
$$


Thanks to $\left|\vec{p}^{*}\right| \in C(\Omega)$, these two set $\Omega_{i}, i=1,2$ are open. In $\Omega_{1}$, we have

$$
\nabla\left|\vec{p}^{*}\right|=\frac{\vec{p}^{*} \cdot \nabla \vec{p}^{*}}{\left|\vec{p}^{*}\right|^{2}}
$$

Therefore

$$
\int_{\Omega_{1}}|\nabla| \vec{p}^{*}||^{p}=\int_{\Omega_{1}} \frac{\left|\vec{p}^{*} \cdot \nabla \vec{p}^{*}\right|^{p}}{\left|\vec{p}^{*}\right|^{2 p}} \leq \frac{\left|\vec{p}^{*}\right|_{L^{\infty}}^{p}}{\left(\frac{\alpha}{2}\right)^{2 p}} \int_{\Omega_{1}}\left|\nabla \vec{p}^{*}\right|^{p}<\infty .
$$

This implies that $\left|\vec{p}^{*}\right| \in W^{1, p}\left(\Omega_{1}\right)$. By $\max (0, \cdot): W^{1, p} \mapsto W^{1, p}$ (see Corollary 6.18 in [10]), and the third equation in (2.9), we obtain $\beta^{*} \in W^{1, p}\left(\Omega_{1}\right)$. On the other hand, $\beta^{*}=\alpha$ in $\Omega_{2}$. Since $\Omega_{i}, i=1,2$ are two open sets, with $\Omega_{1} \cap \Omega_{2} \neq \varnothing$, $\operatorname{dist}\left(\partial \Omega_{1}, \partial \Omega_{2}\right)>0$ and $\Omega_{1} \cup \Omega_{2}=\Omega$ we find

$$
\beta^{*} \in W^{1, p}\left(\Omega_{1} \cup \Omega_{2}\right)=W^{1, p}(\Omega) .
$$

Using $\vec{u}^{*}=\frac{\vec{p}^{*}}{\beta^{*}}$, we find

$$
\begin{aligned}
\int_{\Omega}\left|\nabla \vec{u}^{*}\right|^{p}= & \int_{\Omega}\left|\frac{\beta^{*} \nabla \vec{p}^{*}-\vec{p}^{*} \otimes \nabla \beta^{*}}{\left(\beta^{*}\right)^{2}}\right|^{p} \leq \frac{2^{p-1}}{\alpha^{2 p}} \int_{\Omega}\left(\left|\beta^{*} \nabla \vec{p}^{*}\right|^{p}+\left|\vec{p}^{*} \otimes \nabla \beta^{*}\right|^{p}\right) \\
& \leq \frac{2^{p-1}\left\|\beta^{*}\right\|_{L}^{p} \infty}{\alpha^{2 p}} \int_{\Omega}\left\|\nabla \vec{p}^{*}\right\|^{p}+\frac{2^{p-1}|\vec{p}|_{L}^{p} \infty}{\alpha^{2 p}} \int_{\Omega}\left\|\nabla \beta^{*}\right\|^{p}<\infty
\end{aligned}
$$

where the tensor product $(\cdot \otimes \cdot)$ for two column vectors is defined by

$$
\vec{v}_{1} \otimes \vec{v}_{2}=\vec{v}_{1} \vec{v}_{2}^{T}
$$

Therefore $\vec{u}^{*} \in\left(W^{1, p}\right)^{m}$.

As a consequence of Corollary 2.4 under additional regularity assumptions on the operator $\Lambda$ (e.g. that its coefficients are $C^{0,1}(\Omega)$ ) and on the boundary of $\Omega$ (e.g. that $\partial \Omega$ is of class $C^{2,1}$ ) the optimal solution enjoys the additional regularity $\vec{y}^{*} \in\left(W^{3, p}\right)^{m}$.

\section{Semismooth Newton Algorithm}

We will use a semismooth Newton method to solve the nonlinear system (2.13). First $\vec{u}^{*}$ can be replaced by $\frac{\vec{p}^{*}}{\beta^{*}}$. Let $x=(\vec{y}, \vec{p}, \beta)^{t}$, and

$$
F(x)=\left(\begin{array}{l}
\Lambda \vec{y}-\frac{\vec{p}}{\beta} \\
\Lambda^{*} \vec{p}+\vec{y}-\vec{y}_{d} \\
\beta-\max (\alpha,|\vec{p}|)
\end{array}\right),
$$

where $\max (\alpha,|\vec{p}|)$ is defined pointwise. Associated to $\vec{p}$ define the active set and inactive set as

$$
\mathcal{A}=\{\mathbf{x}:|\vec{p}(\mathbf{x})|>\alpha\}, \quad \mathcal{I}=\{\mathbf{x}:|\vec{p}(\mathbf{x})| \leq \alpha\} .
$$

The active set we defined here is different from the previous definition (2.10) which was used to in proof of Theorem 2.2. The Newton derivative of $F$ at $x$ can be written as follows, see e.g. [5]

$$
D_{N} F(x)=\left(\begin{array}{ccc}
\Lambda & -\frac{1}{\beta} & \frac{\vec{p}}{\beta^{2}} \\
I & \Lambda^{*} & 0 \\
0 & -\frac{\vec{p}^{t}}{|\vec{p}|} \chi_{\mathcal{A}} & I
\end{array}\right),
$$


where $\chi_{\mathcal{A}}$ is a characteristic function. Now we introduce the semismooth Newton iteration from $x^{k}$ to $x^{k+1}$. Define the active sets and inactive sets at iterative level $k$ as

$$
\mathcal{A}^{k}=\left\{\mathbf{x}:\left|\vec{p}^{k}(\mathbf{x})\right|>\alpha\right\}, \quad \mathcal{I}^{k}=\left\{\mathbf{x}:\left|\vec{p}^{k}(\mathbf{x})\right| \leq \alpha\right\} .
$$

Then the Newton step

$$
D_{N} F\left(x^{k}\right)\left(x^{k+1}-x^{k}\right)=-F\left(x^{k}\right)
$$

is equivalent to the system of equations,

$$
\left\{\begin{array}{l}
\Lambda \vec{y}^{k+1}=\frac{1}{\beta^{k}} \vec{p}^{k+1}+\frac{\vec{p}^{k}}{\left(\beta^{k}\right)^{2}}\left(\beta^{k}-\beta^{k+1}\right), \\
\Lambda^{*} \vec{p}^{k+1}=\vec{y}_{d}-\vec{y}^{k+1}, \\
\beta^{k+1}=\alpha \chi_{\mathcal{I}^{k}}+\frac{\vec{p}^{k} \cdot \vec{p}^{k+1}}{\left|\vec{p}^{k}\right|} \chi_{\mathcal{A}^{k}} .
\end{array}\right.
$$

Existence of solution to (3.5) under the assumption that $\left(\beta^{k}, \vec{p}^{k}\right)$ is sufficiently close to $\left(\beta^{*}, \vec{p}^{*}\right)$ will be argued in Theorem 3.5 below.

We give the semismooth Newton algorithm in Algorithm 1. Step 4 ensures that $\beta^{k} \geq \alpha$, and hence the right hand side of first equation in (3.5) is well-defined.

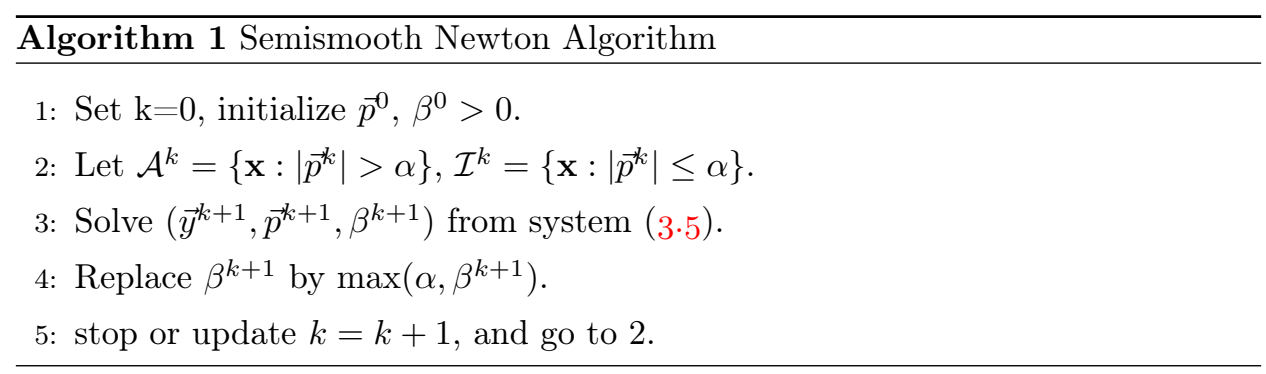

Next we will prove the superlinear convergence of Algorithm 1. For this purpose we introduce

$$
\left(\vec{z}^{k}, \vec{q}^{k}, \gamma^{k}\right)=\left(\vec{y}^{k}, \vec{p}^{k}, \beta^{k}\right)-\left(\vec{y}^{*}, \vec{p}^{*}, \beta^{*}\right) .
$$

This quantity satisfies the error equation

$$
\left\{\begin{array}{l}
\Lambda \vec{z}^{k+1}=\frac{\beta^{*} \vec{q}^{k+1}}{\beta^{*} \beta^{k}}-\vec{p}^{*} \gamma^{k}+\frac{\vec{p}^{k}}{\left(\beta^{k}\right)^{2}}\left(\gamma^{k}-\gamma^{k+1}\right), \\
\Lambda^{*} \vec{q}^{k+1}=-\vec{z}^{k+1}, \\
\gamma^{k+1}=\frac{\vec{p}^{k} \cdot \vec{q}^{k+1}}{\left|\vec{p}^{k}\right|} \chi_{\mathcal{A}^{k}}+R^{k},
\end{array}\right.
$$

where

$$
R^{k}=\max \left(\alpha,\left|\vec{p}^{k}\right|\right)-\max \left(\alpha,\left|\vec{p}^{*}\right|\right)-\frac{\vec{p}^{k} \cdot \vec{q}^{k}}{\left|\vec{p}^{k}\right|} \chi_{\mathcal{A}^{k}} .
$$

By the chain rule, $\vec{p} \rightarrow \max (\alpha,|\vec{p}|)$ is Newton differentiable from $L^{\infty}$ to $L^{p}$, for any $1 \leq p<\infty$ (c.f. [5]), and $D_{N} \max (\alpha,|\vec{p}|)=\frac{\vec{p}}{|\vec{p}|} \chi_{\mathcal{A}}$. Hence

$$
\left\|R^{k}\right\|_{L^{p}}=o\left(\left\|\vec{q}^{k}\right\|_{L^{\infty}}\right) .
$$

Let us briefly outline the following arguments. We start with the technical Lemma 3.1. The Newton iteration (3.5) which is suggested by the structure of the error equation (3.6). Then in Lemmas 3.2 and 3.3 it is verified that the assumption of Lemma 3.1 are applicable for (3.6) so that we obtain the appropriate a-priori 
estimate which allows us to prove wellposedness and superlinear convergence in Theorem 3.5.

Lemma 3.1. Consider the elliptic system with zero Dirichlet boundary condition as

$$
\left\{\begin{array}{l}
\Lambda \vec{z}=(A+B) \vec{q}+\vec{h} \\
\Lambda^{*} \vec{q}=-\vec{z}
\end{array}\right.
$$

Let $A$ be a nonnegative linear operator in $\mathcal{L}\left(\left(L^{2}\right)^{m},\left(L^{2}\right)^{m}\right)$, i.e. $(\vec{q}, A \vec{q}) \geq 0$, for all $\vec{q} \in\left(L^{2}(\Omega)\right)^{m}$, and let $B \in \mathcal{L}\left(\left(L^{2}\right)^{m},\left(L^{2}\right)^{m}\right)$ be a perturbation operator which satisfies $|(\vec{q}, B \vec{q})| \leq \frac{1}{2 \kappa}\|\vec{q}\|_{L^{\infty}}^{2}$, where $\kappa$ is a constant such that

$$
\|u\|^{2}+\|u\|_{L^{\infty}}^{2} \leq \kappa\left\|\Lambda^{*} u\right\|^{2}, \quad \forall u \in H^{2} \cap H_{0}^{1} .
$$

Then system (3.7) has a unique solution $(\vec{z}, \vec{q})$ for any $h \in\left(L^{2}(\Omega)\right)^{m}$, and we have the a-priori estimate

$$
\|\vec{q}\|_{2}+\|\vec{z}\| \leq C\|\vec{h}\|
$$

for a constant $C$ independent of $\vec{h}$.

Proof. We only need to check inequality (3.9), the existence being guaranteed by the Fredholm alternative (c.f. Theorem 5.11 in [3] ). Taking the inner product of (3.7) with $\vec{q}$ we have

$$
(A \vec{q}, \vec{q})+(B \vec{q}, \vec{q})+(\vec{h}, \vec{q})=(\Lambda \vec{z}, \vec{q})=-\left\|\Lambda^{*} \vec{q}\right\|^{2},
$$

and hence

$\left\|\Lambda^{*} \vec{q}\right\|^{2}+(A \vec{q}, \vec{q})=-(B \vec{q}+\vec{h}, \vec{q}) \leq \frac{1}{2 \kappa}\|\vec{q}\|_{L^{\infty}}^{2}+\frac{\kappa}{2}\|\vec{h}\|^{2}+\frac{1}{2 \kappa}\|\vec{q}\|^{2} \leq \frac{1}{2}\left\|\Lambda^{*} \vec{q}\right\|^{2}+\frac{\kappa}{2}\|\vec{h}\|^{2}$.

Together with $\|\vec{q}\|_{2} \leq C\left\|\Lambda^{*} \vec{q}\right\|$, this gives estimate (3.9).

We now return to the error equation. From the first and third equations of (3.6), we have

$\Lambda \vec{z}^{k+1}=\frac{1}{\beta^{k}}\left[\vec{q}^{k+1}-\frac{1}{\beta^{k}}\left(\frac{\overrightarrow{p^{k}} \cdot q^{k+1}}{\left|\overrightarrow{p^{k}}\right|} \chi_{\mathcal{A}^{k}}+R^{k}\right) \vec{p}^{k}+\frac{\gamma^{k}}{\beta^{k}} \vec{p}^{k}-\frac{\gamma^{k}}{\beta^{*}} p^{*}\right]=M^{k} \vec{q}^{k+1}+h^{k}$,

where, using $\gamma^{k}=\beta^{k}-\beta^{*}, \vec{q}^{k}=\vec{p}^{k}-\vec{p}^{*}$,

$$
M^{k}=\frac{1}{\beta^{k}}\left(I-\frac{1}{\beta^{k}} \frac{\vec{p}^{k} \otimes \vec{p}^{k}}{\left|\vec{p}^{k}\right|} \chi_{\mathcal{A}^{k}}\right), \quad h^{k}=\frac{1}{\beta^{k}}\left(\frac{\gamma^{k}}{\beta^{k} \beta^{*}}\left(\beta^{*} \vec{q}^{k}-\gamma^{k} \vec{p}^{*}\right)-\frac{R^{k}}{\beta^{k}} \vec{p}^{k}\right) .
$$

Then we decompose $M^{k}$ into two parts

$$
M^{k}=\underbrace{\frac{1}{\beta^{k}}\left(I-\frac{1}{\beta^{*}} \mathcal{H}\left(\vec{p}^{*}\right) \chi_{\mathcal{A}^{k}}\right)}_{D^{k}}+\underbrace{\frac{1}{\beta^{k}}\left(\frac{1}{\beta^{*}} \mathcal{H}\left(\vec{p}^{*}\right) \chi_{\mathcal{A}^{k}}-\frac{1}{\beta^{k}} \frac{\vec{p}^{k} \otimes \vec{p}^{k}}{\left|\vec{p}^{k}\right|} \chi_{\mathcal{A}^{k}}\right)}_{E^{k}}
$$

where

$$
\mathcal{H}\left(\vec{p}^{*}\right)= \begin{cases}\frac{\vec{p}^{*} \otimes \vec{p}^{*}}{\left|\vec{p}^{*}\right|} & \text { if }\left|\vec{p}^{*}\right|>0 \\ 0_{m \times m} & \text { if } \vec{p}^{*}=0\end{cases}
$$


Lemma 3.2. If $\beta^{k} \geq \alpha$, then $D^{k}$ is a nonnegative operator in $\mathcal{L}\left(\left(L^{2}\right)^{m},\left(L^{2}\right)^{m}\right)$, i.e. $\left(\vec{q}, D^{k} \vec{q}\right) \geq 0$ for all $\vec{q} \in\left(L^{2}(\Omega)\right)^{m}$. Moreover if $\left\|\vec{p}^{k}-\vec{p}^{*}\right\|_{L^{1}}+\left\|\beta^{k}-\beta^{*}\right\|_{L^{1}} \leq \epsilon$, for sufficient small $\epsilon$, then we have

$$
\left|\left(\vec{q}, E^{k} \vec{q}\right)\right| \leq \frac{1}{2 \kappa}\|\vec{q}\|_{L^{\infty}}^{2} .
$$

Proof. We first check that $D^{k}$ is nonnegative. From the optimality system (2.9), we find $\vec{p}^{*}=0 \Leftrightarrow \vec{u}^{*}=0$. Then defining

$$
\mathcal{Z}=\left\{\mathbf{x}:\left|\vec{p}^{*}(\mathbf{x})\right|>0\right\}
$$

we have

$$
\frac{1}{\beta^{*}} \mathcal{H}\left(\vec{p}^{*}\right) \chi_{\mathcal{A}^{k}}=\frac{1}{\beta^{*}} \frac{\vec{p}^{*} \otimes \vec{p}^{*}}{\left|\vec{p}^{*}\right|} \chi_{\mathcal{Z}} \chi_{\mathcal{A}^{k}}=\frac{\vec{u}^{*} \otimes \vec{u}^{*}}{\left|\vec{u}^{*}\right|} \chi_{\mathcal{Z}} \chi_{\mathcal{A}^{k}}, \text { a.e. in } \Omega .
$$

Hence for any $\vec{q} \in\left(L^{2}\right)^{m}$, we obtain

$$
\left(\vec{q}, D^{k} \vec{q}\right)=\left(\vec{q}, \frac{\vec{q}}{\beta^{k}}\right)-\left(\frac{\vec{q}}{\beta^{k}}, \frac{\vec{u}^{*} \otimes \vec{u}^{*}}{\left|\vec{u}^{*}\right|} \chi_{\mathcal{Z}} \chi_{\mathcal{A}^{k}} \vec{q}\right) .
$$

Let $\vec{w}=\frac{\vec{q}}{\sqrt{\beta^{k}}}$ and compute

$$
\left(\vec{q}, D^{k} \vec{q}\right)=\|\vec{w}\|^{2}-\int_{\Omega} \frac{\left(\vec{w} \cdot \vec{u}^{*}\right)^{2}}{\left|\vec{u}^{*}\right|} \chi_{\mathcal{Z}} \chi_{\mathcal{A}^{k}} d x \geq\|\vec{w}\|^{2}-\int_{\Omega}|\vec{w}|^{2}\left|\vec{u}^{*}\right| d x \geq 0 .
$$

The last step is due to the constraint $\left|\vec{u}^{*}\right| \leq 1$. Next we check that $E^{k}$ satisfies (3.13). By (3.14),

$$
\begin{aligned}
& E^{k}=\frac{1}{\beta^{k} \beta^{*}} \frac{\vec{p}^{*} \otimes \vec{p}^{*}}{\left|\vec{p}^{*}\right|} \chi_{\mathcal{Z}} \chi_{\mathcal{A}^{k}}-\frac{1}{\left(\beta^{k}\right)^{2}} \frac{\vec{p}^{k} \otimes \vec{p}^{k}}{\left|\vec{p}^{k}\right|} \chi_{\mathcal{A}^{k}} \\
& =\left(\frac{1}{\beta^{k} \beta^{*}} \frac{\vec{p}^{*} \otimes \vec{p}^{*}}{\left|\vec{p}^{*}\right|}-\frac{1}{\left(\beta^{k}\right)^{2}} \frac{\vec{p}^{k} \otimes \overrightarrow{p^{k}}}{\left|\vec{p}^{k}\right|}\right) \chi_{\mathcal{Z} \cap \mathcal{A}^{k}}-\frac{1}{\left(\beta^{k}\right)^{2}} \frac{\vec{p}^{k} \otimes \vec{p}^{k}}{\left|\vec{p}^{k}\right|} \chi_{\mathcal{A}^{k} \backslash \mathcal{Z}} \\
& =\underbrace{\frac{\left(\left|\vec{p}^{k}\right|-\left|\vec{p}^{*}\right|\right) \vec{p}^{*} \otimes \vec{p}^{*}}{\beta^{k} \beta^{*}\left|\vec{p}^{k}\right|\left|\vec{p}^{*}\right|} \chi_{\mathcal{Z} \cap \mathcal{A}^{k}}}_{N_{1}}+\underbrace{\frac{\left(\beta^{k}-\beta^{*}\right) \vec{p}^{*} \otimes \vec{p}^{*}}{\left(\beta^{k}\right)^{2} \beta^{*}\left|\vec{p}^{k}\right|} \chi_{\mathcal{Z} \cap \mathcal{A}^{k}}}_{N_{2}}+\underbrace{\frac{\left(\vec{p}^{*}-\vec{p}^{k}\right) \otimes \vec{p}^{*}}{\left(\beta^{k}\right)^{2}\left|\vec{p}^{k}\right|} \chi_{\mathcal{Z} \cap \mathcal{A}^{k}}}_{N_{3}} \\
& +\underbrace{\frac{\vec{p}^{k} \otimes\left(\vec{p}^{*}-\vec{p}^{k}\right)}{\left(\beta^{k}\right)^{2}\left|\vec{p}^{k}\right|} \chi_{\mathcal{Z} \cap \mathcal{A}^{k}}}_{N_{4}}-\underbrace{\frac{1}{\left(\beta^{k}\right)^{2}} \frac{\vec{p}^{k} \otimes \vec{p}^{k}}{\left|\vec{p}^{k}\right|} \chi_{\mathcal{A}^{k} \backslash \mathcal{Z}}}_{N_{5}}
\end{aligned}
$$

hence

$$
\left|\left(\vec{q}, E^{k} \vec{q}\right)\right| \leq\left|\left(\vec{q}, N_{1} \vec{q}\right)\right|+\left|\left(\vec{q}, N_{2} \vec{q}\right)\right|+\left|\left(\vec{q}, N_{3} \vec{q}\right)\right|+\left|\left(\vec{q}, N_{4} \vec{q}\right)\right|+\left|\left(\vec{q}, N_{5} \vec{q}\right)\right| .
$$


We need to estimate each term:

$$
\begin{aligned}
& \left|\left(\vec{q}, N_{1} \vec{q}\right)\right| \leq \frac{1}{\alpha^{2}} \int_{\Omega} \frac{\left(\left|\vec{p}^{k}-\vec{p}^{*}\right|\right)\left|\vec{p}^{*}\right|}{\left|\vec{p}^{k}\right|}|\vec{q}|^{2} \chi_{\mathcal{Z} \cap \mathcal{A}^{k}} d x \leq \frac{1}{\alpha^{3}}\left\|\vec{p}^{*}\right\|_{L^{\infty}}\left\|\vec{p}^{k}-\vec{p}^{*}\right\|_{L^{1}}\|\vec{q}\|_{L^{\infty}}^{2} \leq C \epsilon\|\vec{q}\|_{L^{\infty}}^{2}, \\
& \left|\left(\vec{q}, N_{2} \vec{q}\right)\right| \leq \frac{1}{\alpha^{3}} \int_{\Omega} \frac{\left|\vec{p}^{*}\right|^{2}}{\left|\vec{p}^{k}\right|}\left|\beta^{k}-\beta^{*}\right||\vec{q}|^{2} \chi_{\mathcal{Z} \cap \mathcal{A}^{k}} d x \leq \frac{C}{\alpha^{4}}\left\|\vec{p}^{*}\right\|_{L^{\infty}}^{2}\left\|\beta^{k}-\beta^{*}\right\|_{L^{1}}\|\vec{q}\|_{L^{\infty}}^{2} \leq C \epsilon\|\vec{q}\|_{L^{\infty}}^{2}, \\
& \left|\left(\vec{q}, N_{3} \vec{q}\right)\right| \leq \frac{1}{\alpha^{2}} \int_{\Omega} \frac{\left(\left|\vec{p}^{k}-\vec{p}^{*}\right|\right)\left|\vec{p}^{*}\right|}{\left|\vec{p}^{k}\right|}|\vec{q}|^{2} \chi_{\mathcal{Z} \cap \mathcal{A}^{k}} d x \leq \frac{1}{\alpha^{3}}\left\|\vec{p}^{*}\right\|_{L^{\infty}}\left\|\vec{p}^{k}-\vec{p}^{*}\right\|_{L^{1}}\|\vec{q}\|_{L^{\infty}}^{2} \leq C \epsilon\|\vec{q}\|_{L^{\infty}}^{2}, \\
& \left|\left(\vec{q}, N_{4} \vec{q}\right)\right| \leq \frac{1}{\alpha^{2}} \int_{\Omega} \frac{\left(\left|\vec{p}^{k}-\vec{p}^{*}\right|\right)\left|\vec{p}^{*}\right|}{\left|\vec{p}^{k}\right|}|\vec{q}|^{2} \chi_{\mathcal{Z} \cap \mathcal{A}^{k}} d x \leq \frac{1}{\alpha^{2}}\left\|\vec{p}^{k}-\vec{p}^{*}\right\|_{L^{1}}\|\vec{q}\|_{L^{\infty}}^{2} \leq C \epsilon\|\vec{q}\|_{L^{\infty}}^{2}, \\
& \left|\left(\vec{q}, N_{5} \vec{q}\right)\right| \leq \frac{1}{\alpha^{2}} \int_{\Omega}\left|\vec{p}^{k}\right||\vec{q}|^{2} \chi_{\mathcal{A}^{k} \backslash \mathcal{Z}} d x \leq \frac{1}{\alpha^{2}}\left\|\vec{p}^{k}-\vec{p}^{*}\right\|_{L^{1}}\|\vec{q}\|_{L^{\infty}}^{2} \leq C \epsilon\|\vec{q}\|_{L^{\infty}}^{2} .
\end{aligned}
$$

The last inequality follows from the fact:

$$
\vec{p}^{*}=\vec{p}^{*}-\vec{p}^{*}, \forall \mathbf{x} \in \mathcal{A}^{k} \backslash \mathcal{Z} .
$$

If $\epsilon$ is sufficiently small, we obtain (3.13).

Lemma 3.3. Let $\beta^{k} \geq \alpha$, and $\left\|\vec{p}^{k}-\vec{p}^{*}\right\|_{L^{4}}+\left\|\beta^{k}-\beta^{*}\right\|_{L^{4}} \leq \epsilon$, then for sufficiently small $\epsilon$, we have $\left\|h^{k}\right\|=o(\epsilon)$.

Proof. Recall the definition (3.11),

$$
h^{k}=\frac{1}{\beta^{k}}\left(\frac{\gamma^{k}}{\beta^{k} \beta^{*}}\left(\beta^{*} \vec{q}^{k}-\gamma^{k} \vec{p}^{*}\right)-\frac{R^{k}}{\beta^{k}} \vec{p}^{k}\right),
$$

and $\gamma^{k}=\beta^{k}-\beta^{*}$. Then

$$
\begin{aligned}
& \left\|\frac{\gamma^{k}}{\left(\beta^{k}\right)^{2}} \vec{q}^{k}\right\| \leq \frac{1}{\alpha^{2}}\left\|\vec{q}^{k}\right\|_{L^{4}}\left\|\gamma^{k}\right\|_{L^{4}}=o(\epsilon), \\
& \left\|\frac{\left(\gamma^{k}\right)^{2}}{\left(\beta^{k}\right)^{2} \beta^{*}} \vec{p}^{*}\right\| \leq \frac{1}{\alpha^{3}}\left\|\vec{p}^{*}\right\|_{L^{\infty}}\left\|\gamma^{k}\right\|_{L^{4}}^{2}=o(\epsilon), \\
& \left\|\frac{R^{k}}{\left(\beta^{k}\right)^{2}} \vec{p}^{k}\right\| \leq \frac{1}{\alpha^{2}}\left\|\vec{p}^{k}\right\|_{L^{4}}\left\|R^{k}\right\|_{L^{4}}=o(\epsilon),
\end{aligned}
$$

which completes the estimate.

Remark 3.4. Since $\Omega$ is bounded domain, the assumption $\left\|\vec{p}^{*}-\vec{p}^{*}\right\|_{L^{4}}+\| \beta^{k}-$ $\beta^{*} \|_{L^{4}} \leq \epsilon$ is stronger than the assumption in Lemma 3.2, and this technical assumption will be used to prove superlinear convergence.

Now we move to our convergence result for the semismooth Newton method.

Theorem 3.5. Superlinear convergence for Algorithm 1.

If $\left\|\beta^{k}-\beta^{*}\right\|_{L^{4}}+\left\|\vec{p}^{k}-\vec{p}^{*}\right\|_{L^{4}} \leq \epsilon$ and $\epsilon>0$ is sufficiently small, then the Newton iteration in Algorithm 1 is well defined and $\left\|\beta^{k+1}-\beta^{*}\right\|_{L^{4}}+\left\|\vec{p}^{k+1}-\vec{p}^{*}\right\|_{\infty}=o(\epsilon)$.

Proof. To prove local wellposedness of the Newton step (3.5), we will use the error equation (3.6). Substituting $\gamma^{k+1}$ into the first equations in (3.6), we obtain equation (3.10). Let

$$
D^{k}=A, \quad E^{k}=B, \quad \vec{z}^{k+1}=\vec{z}, \quad \vec{q}^{k+1}=\vec{q}, \quad \vec{h}^{k}=\vec{h},
$$

where $D^{k}, E^{k}$ and $h^{k}$ are defined in (3.12) and (3.11). By Lemma 3.2, it is noticed that $D^{k}$ and $E^{k}$ satisfy the conditions in Lemma 3.1. Therefore, by Lemma 3.1, the 
error equation (3.6) admits a unique solution if $\epsilon$ is sufficiently small. This implies that $(3.5)$ admits a unique solution $\left(\vec{y}^{k+1}, \vec{p}^{k+1}, \tilde{\beta}^{k+1}\right)$, if $\left\|\beta^{k}-\beta^{*}\right\|_{L^{4}}+\left\|\vec{p}^{k}-\vec{p}^{*}\right\|_{L^{4}}$ is sufficiently small.

We next argue superlinear convergence. Combining Lemma 3.1 and Lemma 3.3 we have

$$
\left\|\vec{p}^{k+1}-\vec{p}^{*}\right\|_{2}+\left\|\vec{y}^{k+1}-\vec{y}^{*}\right\| \leq C\left\|\vec{h}^{k}\right\|=o(\epsilon) .
$$

In particular this also implies that $\left\|\vec{p}^{k+1}-\vec{p}^{*}\right\|_{L^{\infty}}=o(\epsilon)$. Then we notice that

$$
\left\|\tilde{\beta}^{k+1}-\beta^{*}\right\|_{L^{4}} \leq\left\|\frac{\vec{p}^{k} \cdot \vec{q}^{k+1}}{\left|\vec{p}^{k}\right|} \chi_{\mathcal{A}^{k}}\right\|_{L^{4}}+\left\|R^{k}\right\|_{L^{4}} \leq C\left\|\vec{p}^{k+1}-\vec{p}^{*}\right\|_{L^{4}}+\left\|R^{k}\right\|_{L^{4}}=o(\epsilon) .
$$

Thanks to $\beta^{*} \geq \alpha$, the projection step does not increase error, i.e.

$$
\left|\beta^{k+1}(\mathbf{x})-\beta^{*}(\mathbf{x})\right|=\left|\max \left(\alpha, \tilde{\beta}^{k+1}(\mathbf{x})\right)-\beta^{*}(\mathbf{x})\right| \leq\left|\tilde{\beta}^{k+1}(\mathbf{x})-\beta^{*}(\mathbf{x})\right| \text {, a.e. in } \Omega \text {. }
$$

Then we obtain

$$
\left\|\beta^{k+1}-\beta^{*}\right\|_{L^{4}}+\left\|\vec{p}^{k+1}-\vec{p}^{*}\right\|_{\infty}=o(\epsilon)
$$

and this concludes the proof.

Remark 3.6. We eliminated the control variable $\vec{u}$ from the optimality system using $\vec{p}=\beta \vec{u}$. The semismooth Newton algorithm can also be applied if we treat $\vec{u}$ as an independent variable.

\section{Generalization}

We will discuss a few generalizations which are related to Problem 1.1. Recall the optimality system we have obtained in Theorem 2.2,

$$
\left\{\begin{array}{l}
\Lambda \vec{y}^{*}=E \vec{u}^{*}, \\
\Lambda^{*} \vec{p}^{*}=\vec{y}_{d}-\vec{y}^{*}, \\
E^{*} \vec{p}^{*}=\alpha \vec{u}^{*}+\lambda^{*} B^{T}\left(B \vec{u}^{*}-\vec{b}\right), \\
\lambda^{*} \geq 0,\left|B \vec{u}^{*}-\vec{b}\right| \leq 1, \lambda^{*} \cdot\left(\left|B \vec{u}^{*}-\vec{b}\right|-1\right)=0 .
\end{array}\right.
$$

To apply the semismooth Newton algorithm in general case, we need to rewrite the optimality system in terms of a system of nonlinear equations which are Newton differentiable. Since the primal and adjoint equations are both linear, we only focus on the optimality and the complementary conditions.

In this section we generalize our previous work in three directions, namely the case where the control $\vec{u}$ is supported in a subdomain $\tilde{\Omega} \subset \Omega$, the case of an arbitrary translation vector $\vec{b}$, and the case of an invertible linear transformation $B$. For simplicity of discussion, we take each generalization individually, but these generalization can be combined.

Our goal in the following subsections is to rewrite the optimality system into equivalent nonlinear equations and to prove that these nonlinear equations are actually Newton differentiable, and hence that the semismooth Newton method provides an efficient algorithm with locally superlinear convergence. 


\subsection{Extension to a control domain $\tilde{\Omega} \subset \Omega$.}

Recall the definition of the bounded linear operator $E:\left(L^{2}(\tilde{\Omega})\right)^{m} \mapsto\left(L^{2}(\Omega)\right)^{m}$ as the extension-by-zero operator and its dual operator $E^{*}:\left(L^{2}(\Omega)\right)^{m} \mapsto\left(L^{2}(\tilde{\Omega})\right)^{m}$ is the restriction operator $E^{*} \vec{f}=\vec{f} \chi_{\tilde{\Omega}}$. Therefore the optimality condition can be equivalently expressed as

$$
\vec{p} \chi_{\tilde{\Omega}}=\alpha \vec{u}+2 \lambda \vec{u} .
$$

Using the similar arguments as in equation (2.13), the optimality and complementary conditions can be expressed in the following nonlinear equation

$$
\vec{p} \chi_{\tilde{\Omega}}=\beta \vec{u}, \quad \beta=\max (\alpha,|\vec{p}|) .
$$

For a given $\vec{y}_{d} \in L^{2}(\Omega)$, the adjoint equation provides a solution $\vec{p} \in H^{2} \cap H_{0}^{1}(\Omega) \hookrightarrow$ $C_{0}(\Omega)$. Then the mapping $\vec{p} \mapsto \max (\alpha,|\vec{p}|)$ is Newton differentiable (c.f. [5]) from $L^{\infty}$ to $L^{p}$, for any $p<\infty$. Hence this nonlinear equation can be solved by a semismooth Newton algorithm as in section 3.

\subsection{Extension to a general translation vector $b$.}

In this case, the optimality system reads

$$
\vec{p}=\alpha \vec{u}+2 \lambda(\vec{u}-\vec{b}),
$$

and it can be equivalently expressed as

$$
\vec{p}-\alpha \vec{b}=(\alpha+2 \lambda)(\vec{u}-\vec{b}) .
$$

We can then rewrite the optimality condition and complementary condition as

$$
\vec{p}-\alpha \vec{b}=\beta \vec{u}, \quad \beta=\max (\alpha,|\vec{p}-\alpha \vec{b}|)
$$

by applying the same argument as in equation (2.13). This is again a Newton differentiable function and the semismooth Newton algorithm is applicable.

The first two generalizations are straightforward, but the next case which involves a general linear transformation $B$, is rather complicated.

\subsection{Extension to a general invertible matrix $B$.}

For a general $m \times m$ invertible matrix $B$, the optimality and the complementary conditions hold in a pointwise a.e. sense as in Theorem 2.2:

$$
\begin{aligned}
& \vec{p}^{*}(\mathbf{x})=\left(\alpha I+\lambda^{*} B^{T} B\right) \vec{u}^{*}(\mathbf{x}), \\
& \lambda^{*}(\mathbf{x}) \geq 0,\left|B \vec{u}^{*}(\mathbf{x})\right| \leq 1, \lambda^{*}(\mathbf{x}) \cdot\left(\left|B \vec{u}^{*}(\mathbf{x})\right|-1\right)=0 .
\end{aligned}
$$

Equation (4.1) is equivalent to

$$
B^{-T} \vec{p}^{*}=\left(\alpha D+\lambda^{*} I\right)\left(B \vec{u}^{*}\right),
$$

where $D=\left(B B^{T}\right)^{-1}$ is a symmetric, positive definite (SPD) matrix. The eigenvalues of $D$ satisfy

$$
0<\xi_{1} \leq \xi_{2} \leq \cdots \leq \xi_{m}<\infty .
$$

Lemma 4.1. Given a nonzero vector $\vec{p}$, the real valued function

$$
h_{\vec{p}}(\lambda): \lambda \mapsto\left|(\alpha D+\lambda I)^{-1} B^{-T} \vec{p}\right|
$$

is well-defined and strictly monotonically decreasing in $\left(-\alpha \xi_{1}, \infty\right)$. Moreover, $\lim _{\lambda \rightarrow \infty} h_{\vec{p}}(\lambda)=$ 0 . 
Proof. First we observe for every $\lambda>-\alpha \xi_{1}$, the matrix $\alpha D+\lambda I$ is also a SPD matrix, hence it is invertible, and the function $h_{\vec{p}}$ is well-defined. Now we check the monotone property. Let

$$
-\alpha \xi_{1}<\lambda_{1}<\lambda_{2}, \quad \vec{q}_{1}=\left(\alpha D+\lambda_{1} I\right)^{-1} B^{-T} \vec{p}, \quad \vec{q}_{2}=\left(\alpha D+\lambda_{2} I\right)^{-1} B^{-T} \vec{p},
$$

and define

$$
\tilde{q}=\left(\alpha D+\lambda_{1} I\right)^{-1}\left(\alpha D+\lambda_{2} I\right)^{-1} B^{-T} \vec{p} .
$$

Clearly $\tilde{q}$ is not the zero vector. Since matrices $\alpha D+\lambda_{1} I$ and $\alpha D+\lambda_{2} I$ commute, we have

Therefore,

$$
\vec{q}_{1}=\left(\alpha D+\lambda_{2} I\right) \tilde{q}, \quad \vec{q}_{2}=\left(\alpha D+\lambda_{1} I\right) \tilde{q}
$$

$$
\begin{aligned}
\left|\vec{q}_{1}\right|^{2} & =\left|\vec{q}_{2}+\left(\lambda_{2}-\lambda_{1}\right) \tilde{q}\right|^{2}=\left|\vec{q}_{2}\right|^{2}+\left(\lambda_{2}-\lambda_{1}\right)^{2}|\tilde{q}|^{2}+\left(\lambda_{2}-\lambda_{1}\right)\left(\vec{q}_{2}, \tilde{q}\right) \\
& >\left|\vec{q}_{2}\right|^{2}+\left(\lambda_{2}-\lambda_{1}\right)\left(\left(\alpha D+\lambda_{1} I\right) \tilde{q}, \tilde{q}\right)>\left|\overrightarrow{q_{2}}\right|^{2},
\end{aligned}
$$

which implies the strict monotonicity. The last claim $\lim _{\lambda \rightarrow \infty} h_{\vec{p}}(\lambda)=0$ is straightforward.

If $h_{\vec{p}}(0)=\left|(\alpha D)^{-1} B^{-T} \vec{p}\right| \geq 1$, then there exists by Lemma 4.1 a unique $\lambda \geq 0$, such that $h_{\vec{p}}(\lambda)=1$. With the help of this fact, we can define a mapping $g: \vec{p} \mapsto \lambda$ as

$(4 \cdot 5)$

$g(\vec{p})= \begin{cases}0 & \text { if }\left|(\alpha D)^{-1} B^{-T} \vec{p}\right|<1, \\ \text { the unique nonnegative solution to } h_{\vec{p}}(\lambda)=1 & \text { if }\left|(\alpha D)^{-1} B^{-T} \vec{p}\right| \geq 1 .\end{cases}$

Lemma 4.2. Using the function $g(\vec{p})$ as above, we can rewrite the optimality and the complementary conditions (4.1) - (4.2) in one equation:

$$
\vec{p}^{*}(\mathbf{x})=\alpha \vec{u}^{*}(\mathbf{x})+g\left(\vec{p}^{*}(\mathbf{x})\right) B^{T} B \vec{u}^{*}(\mathbf{x}) \text {, a.e. in } \Omega .
$$

Proof. Throughout the proof we evaluate the operators at a fixed point $\mathbf{x} \in \Omega$ which is not indicated in the notation. We first assume that $(4.1)-(4.2)$ hold. If $h_{\vec{p}^{*}}(0)=\left|(\alpha D)^{-1} B^{-T} \vec{p}^{*}\right|<1$, we have $\left|B \vec{u}^{*}\right|=h_{\vec{p}^{*}}\left(\lambda^{*}\right) \leq h_{\vec{p}^{*}}(0)<1$ and hence $\lambda^{*}=0=g\left(\vec{p}^{*}\right)$. On the other hand, if $h_{\vec{p}^{*}}(0)=\left|(\alpha D)^{-1} B^{-T} \vec{p}^{*}\right| \geq 1$, then a proof by contradiction implies that it must be an active point, i.e., $\left|B \vec{u}^{*}\right|=1$. To see that, we assume this point is not active, i.e. $\left|B \vec{u}^{*}\right|<1$. By the complementary condition (4.2), $\lambda^{*}=0$. Hence $\left|B \vec{u}^{*}\right|=h_{\vec{p}^{*}}\left(\lambda^{*}\right) \geq 1$ which is a contradiction.

In this case, $\lambda^{*}$ satisfies $h_{\vec{p}^{*}}\left(\lambda^{*}\right)=1$, and hence $\lambda^{*}=g\left(\vec{p}^{*}\right)$.

Conversely, if

$$
\vec{p}^{*}=\alpha \vec{u}^{*}+g\left(\vec{p}^{*}\right) B^{T} B \vec{u}^{*},
$$

we can then define $\lambda^{*}=g\left(\vec{p}^{*}\right)$. Equation (4.1) is clearly satisfied. For the complementary condition (4.2), we only need to check $\lambda^{*}\left(\left|B \vec{u}^{*}\right|-1\right)=0$. Suppose $\lambda^{*}=g\left(\vec{p}^{*}\right)>0$. From $(4.5)$ we have

$$
\left|(\alpha D)^{-1} B^{-T} \vec{p}^{*}\right| \geq 1 \text { and } h_{\vec{p}^{*}}\left(\lambda^{*}\right)=1 .
$$

The later equality implies by $(4 \cdot 3)$ that

$$
1=h_{\vec{p}^{*}}\left(\lambda^{*}\right)=\left|\left(\alpha D+\lambda^{*} I\right)^{-1} B^{-T} \vec{p}^{*}\right|=\left|B \vec{u}^{*}\right| .
$$


By singular value decomposition, we can express $D$ as

$$
D=Q^{-1} \hat{D} Q
$$

where $Q$ is an orthonormal matrix and $\hat{D}$ is a diagonal matrix of the form $\operatorname{diag}\left(\xi_{1}, \xi_{2}, \ldots, \xi_{m}\right)$.

Then the equation

$$
\left|(\alpha D+\lambda I)^{-1} B^{-T} \vec{p}\right|=1
$$

can be simplified as

$$
\left|(\alpha \hat{D}+\lambda I)^{-1} Q B^{-T} \vec{p}\right|=1
$$

Let

$$
\hat{p}=Q B^{-T} \vec{p}=\left(\hat{p}_{1}, \ldots, \hat{p}_{m}\right) .
$$

Then equation $\left|(\alpha \hat{D}+\lambda I)^{-1} Q B^{-T} \vec{p}\right|=1$ becomes

$$
\frac{\hat{p}_{1}^{2}}{\left(\alpha \xi_{1}+\lambda\right)^{2}}+\cdots+\frac{\hat{p}_{m}^{2}}{\left(\alpha \xi_{m}+\lambda\right)^{2}}=1
$$

We define the set

$$
\mathcal{O}=\left\{\vec{q} \in \mathbb{R}^{m}:\left|(\alpha \hat{D}+\lambda I)^{-1} \vec{q}\right|=1, \text { for some } \lambda>0\right\}
$$

and observe that it can be equivalently expressed as

$$
\mathcal{O}=\left\{\vec{q} \in \mathbb{R}^{m}:\left|(\alpha \hat{D})^{-1} \vec{q}\right|>1\right\} .
$$

This follows from the fact that $\lambda \rightarrow\left|(\alpha \hat{D}+\lambda I)^{-1} \vec{q}\right|$, with $\vec{q} \in \mathbb{R}^{m}$, is strictly monotonically increasing on $\left(-\alpha \xi_{1}, \infty\right)$, which can be argued as in the proof of Lemma 4.1. We further define $\mathcal{F}: \mathcal{O} \rightarrow(0, \infty)$ by

$$
\lambda \in \mathcal{F}(\vec{q}) \Leftrightarrow\left|(\alpha \hat{D}+\lambda I)^{-1} \vec{q}\right|=1
$$

Lemma 4.3. The following properties hold.

(1) The map $\mathcal{F}$ is a well defined single valued function.

(2) $\mathcal{O}$ is open, and the function $\mathcal{F}$ is continuously differentiable in $\mathcal{O}$.

Proof. (1) By definition of $\mathcal{O}$, for any $\vec{p} \in \mathcal{O}, \mathcal{F}(\vec{p})$ is not empty. We notice that $\overrightarrow{0} \notin \mathcal{O}$. Since $\lambda \mapsto\left|(\alpha \hat{D}+2 \lambda I)^{-1} \vec{p}\right|$ is strictly monotonically decreasing from $(0, \infty)$ to $\mathbb{R}$, the operation $\mathcal{F}$ is a single valued.

(2) Let $G: \mathbb{R}^{m} \times(0, \infty) \mapsto \mathbb{R}$ be given by

$$
G\left(q_{1}, \ldots, q_{m}, \lambda\right)=\frac{q_{1}^{2}}{\left(\alpha \xi_{1}+2 \lambda\right)^{2}}+\cdots+\frac{q_{m}^{2}}{\left(\alpha \xi_{m}+2 \lambda\right)^{2}}-1 .
$$

Then $G$ is a smooth function in the domain $\mathbb{R}^{m} \times(0, \infty)$. At any point $\vec{p}=$ $\left(p_{1}, \ldots, p_{m}\right) \in \mathcal{O}$, there exists a unique $\lambda \in(0, \infty)$, such that $\lambda=\mathcal{F}(\vec{p})$. This implies that $G\left(p_{1}, \ldots, p_{m}, \lambda\right)=0$, and

$$
\frac{\partial G}{\partial \lambda}=-2\left(\frac{p_{1}^{2}}{\left(\alpha \xi_{1}+2 \lambda\right)^{3}}+\cdots+\frac{p_{m}^{2}}{\left(\alpha \xi_{m}+2 \lambda\right)^{3}}\right)<0 .
$$

By the implicit function theorem, (c.f. pp.224 in [13]): there exists an open neighborhood $U(\vec{p}) \subset \mathbb{R}^{m}$, such that for any $\vec{q} \in U(\vec{p})$, we can find a unique $\tau \in(0, \infty)$, such that $G(\vec{q}, \tau)=0$. This defines a smooth map $\tau=\tau(\vec{q})$ from $U(\vec{p})$ to $\mathbb{R}$.

From this result, we conclude that $U(\vec{p}) \subset \mathcal{O}$, which implies that the set $\mathcal{O}$ is open. From (1) we find that $\tau=\mathcal{F}(\vec{q})$ for any $\vec{q} \in U(\vec{p})$. Therefore, the function $\mathcal{F}$ is continuously differentiable in $\mathcal{O}$. 
We next extend $\mathcal{F}$ from $\mathcal{O}$ to all of $\mathbb{R}^{m}$ by setting

$$
\tilde{\mathcal{F}}(\vec{q})=\left\{\begin{aligned}
\mathcal{F}(\vec{q}) & \text { for } \vec{q} \in \mathcal{O} \\
0 & \text { for } \vec{q} \in \mathcal{O}^{c} .
\end{aligned}\right.
$$

From (4.7) it follows that $\mathcal{O}^{c}$ is a closed convex neighborhood of the origin.

Lemma 4.4. The mapping $\mathcal{F}: \mathbb{R}^{m} \rightarrow \mathbb{R}^{1}$ is semismooth.

Proof. The set of concern is the boundary $\Gamma$ of $\mathcal{O}$. On this set $\tilde{\mathcal{F}}$ is assigned the value 0 . Moreover by construction, if $q_{n} \rightarrow q$ with $q_{n} \in \mathcal{O}$ and $q \in \Gamma$ then $\lim _{q_{n} \rightarrow q} \mathcal{F}\left(q_{n}\right)=0$, and hence $\mathcal{F}$ is continuous on $\mathbb{R}^{m}$.

Next we characterize the derivative of $\vec{q} \rightarrow \lambda(\vec{q})$ at some $\vec{q} \in \operatorname{int} \mathcal{O}$. We observe that $\mathcal{F}(\vec{q})=\lambda$ is equivalent to $\left|(\alpha \hat{D}+\lambda I)^{-1} \vec{q}\right|^{2}=1$. Let $\tilde{\mathcal{F}}^{\prime}=\tilde{\mathcal{F}}^{\prime}(\vec{q}) \vec{h}$ denote the derivative of $\lambda$ at $\vec{q}$ in direction $\vec{h}$ and set

$$
\mathcal{E}=\alpha \hat{D}+\lambda I, \text { where } \lambda=\lambda(\vec{q}) .
$$

Then

$$
\left(\mathcal{E}^{-1} \vec{q}, \mathcal{E}^{-1} \tilde{\mathcal{F}}^{\prime} \mathcal{E}^{-1} \vec{q}\right)_{\mathbb{R}^{m}}=\left(\mathcal{E}^{-1} \vec{q}, \mathcal{E}^{-1} \vec{h}\right)_{\mathbb{R}^{m}}
$$

and hence

$$
\tilde{\mathcal{F}}^{\prime}=\tilde{\mathcal{F}}^{\prime}(\vec{q}) \vec{h}=\frac{1}{\left(\mathcal{E}^{-1} \vec{q}, \mathcal{E}^{-2} \vec{q}\right)_{\mathbb{R}^{m}}}\left(\mathcal{E}^{-1} \vec{q}, \mathcal{E}^{-1} \vec{h}\right)_{R^{m}}, \text { for } \vec{q} \in \mathcal{O} .
$$

For $\vec{q} \in \Gamma$, expression (4.9) provides the directional derivative into the exterior of $\mathcal{O}^{c}$. Since $\tilde{\mathcal{F}}(\vec{q})=0$ for $\vec{q} \in \Gamma$ we find

$\left(\tilde{\mathcal{F}}^{\prime}\right)^{+}=\tilde{\mathcal{F}}^{\prime}(\vec{q})\left(\vec{q}_{e}-\vec{q}\right)=\frac{1}{\left|(\alpha \hat{D})^{-\frac{3}{2}} \vec{q}\right|^{2}}\left((\alpha \hat{D})^{-1} \vec{q},(\alpha \hat{D})^{-1}\left(\vec{q}_{e}-\vec{q}\right)\right)_{\mathbb{R}^{m}}, \vec{q}_{e} \in \mathcal{O}, \vec{q} \in \Gamma$,

where $\left(\tilde{\mathcal{F}}^{\prime}\right)^{+}$denotes the directional derivative of $\tilde{\mathcal{F}}$ at $\vec{q} \in \Gamma$ into the exterior of $\mathcal{O}^{c}$, whereas the directional derivative $(\tilde{\mathcal{F}})^{-}$at $\vec{q} \in \Gamma$ into the interior of $\mathcal{O}^{c}$ is clearly 0 . From this discussion and $(4.9),(4.10)$ we also conclude that $\tilde{\mathcal{F}}$ is locally Lipschitz continuous on $\mathbb{R}^{m}$.

We next argue that $\tilde{\mathcal{F}}$ is globally Lipschitz continuous on $\mathbb{R}^{m}$. In view of (4.9), (4.10) and since $\tilde{\mathcal{F}}^{\prime}=0$ in the interior of $\mathcal{O}^{c}$ it suffices obtain a bound on the operator norm of $\tilde{\mathcal{F}}^{\prime}(q)$ for $q \rightarrow \infty$. For this purpose observe from (4.6) that asymptotically for $|q|_{\mathbb{R}^{m}} \rightarrow \infty$ we have that $\lambda(q)$ behaves like $|q|_{R^{m}}$. Together with the fact that there exists a constant $k_{1}$ independent of $q$ and $\lambda$ such that

$$
\lambda(q)|q| \leq|E q|=|(\alpha \hat{D}+\lambda(q) I) q| \leq k_{1}(\alpha+\lambda(q))|q|
$$

this implies that

$$
\frac{1}{\left(E^{-1} \vec{q}, E^{-2} \vec{q}\right)_{\mathbb{R}^{m}}} \sim|q|_{\mathbb{R}^{m}} \text { for }|q|_{\mathbb{R}^{m}} \rightarrow \infty .
$$

But $\left(E^{-1} \vec{q}, E^{-2} \vec{h}\right)_{\mathbb{R}^{m}} \leq \frac{|q|}{\lambda(q)^{2}}|\vec{h}| \sim \frac{|\vec{h}|}{|q| \mathbb{R}^{m}}$. Combined with (4.9) and (4.11) this implies global Lipschitz continuity of $\tilde{\mathcal{F}}$ from $\mathbb{R}^{m}$ to $\mathbb{R}$. To ascertain semismoothness of $\tilde{\mathcal{F}}$ it now suffices to show that at every $\vec{q} \in \mathbb{R}^{m}$

$$
\lim _{\vec{q}+\vec{h} \in D_{\tilde{\mathcal{F}}},|\vec{h}|_{\mathbb{R}} m \rightarrow 0} \frac{1}{\vec{h}}\left(\tilde{\mathcal{F}}^{\prime}(\vec{q}+\vec{h}) \vec{h}-\tilde{\mathcal{F}}^{\prime}(\vec{q}) \vec{h}\right)=0,
$$


[8], pg.218, where $D_{\tilde{\mathcal{F}}} \subset \mathbb{R}^{m}$ denotes the set where $\tilde{\mathcal{F}}$ is differentiable, which in our case is $\mathbb{R}^{m} \backslash \Gamma$ and $\tilde{\mathcal{F}}^{\prime}$ denotes the directional derivative of $\tilde{\mathcal{F}}$. Property (4.12) follows from the fact that $\tilde{\mathcal{F}}$ is $C^{2}$ in $\mathcal{O}$ as well as in $\mathcal{O}^{c} \backslash \Gamma$. This concludes the proof.

Now we can characterize the map $g(\vec{p}): \vec{p} \mapsto \lambda$ by

$$
g(\vec{p})=Q^{-1} \tilde{\mathcal{F}}\left(Q B^{-T} \vec{p}\right),
$$

where $\tilde{\mathcal{F}}$ is defined in $(4.8)$.

From Lemma 4.4 and the techniques utilized in its proof it follows that $g$ : $\mathbb{R}^{m} \rightarrow \mathbb{R}^{1}$ is semismooth. This mapping induces in a canonical way a substitution operator from $\left(L^{p_{1}}(\Omega)\right)^{m}$ to $L^{p_{2}}(\Omega)$ which we denote by the same symbol. The following result addresses its semismoothness.

Proposition 4.5. If $1 \leq p_{2}<p_{1} \leq \infty$, then the substitution operator associated to $g$ considered as mapping from $\left(L^{p_{1}}(\Omega)\right)^{m}$ to $L^{p_{2}}(\Omega)$ is semismooth.

Proof. This follows from well-known results on substitution operators for semismooth functions, see [15], or [16], pg. 56, 61, or [8], pg. 235.

The optimality and complementarity involving a general matrix B can therefore be expressed as

$$
\vec{p}^{*}=\alpha \vec{u}^{*}+g\left(\vec{p}^{*}\right) B^{T} B \vec{u}^{*},
$$

where the semismooth function $g(\vec{p})$ is defined as in (4.5)

Here $\vec{p}$ can be considered as independent variable, satisfying primal and adjoint equations, or as dependent variable, considered as function of $\vec{u}^{*} \in\left(L^{2}(\Omega)\right)^{m}$. In any case under appropriate regularity condition on $\partial \Omega$ and the coefficients of $\Lambda$, $\vec{p}$ can be considered as element of $\left(H^{2}(\Omega) \cap H_{0}^{1}(\Omega)\right)^{m}$. Considering the product structure involving $g\left(\vec{p}^{*}\right)$ and $u^{*}$, equation (4.14) is a semismooth equation in (at least) $L^{1}(\Omega)$. Even though the function $g(\vec{p})$ is well defined and serves for verifying the local superlinear convergence of the semismooth Newton method, it may be difficult to realize it in practice since we need to solve the $2 m$-order polynomial equation in the algorithm,

$$
\frac{p_{1}^{2}}{\left(\xi_{1}+\lambda\right)^{2}}+\cdots+\frac{p_{m}^{2}}{\left(\xi_{m}+\lambda\right)^{2}}=1
$$

When $m=2$, this can be solved explicitly. If $m \geq 3$, the explicit solution does not exist in general. Therefore the implementation is still a challenging problem.

\section{NUMERICAL TEST}

Here we validate the superlinear convergence for a few simple test problems. The first and second numerical examples involve the vector Laplace equations on the unit square $[0,1] \times[0,1]$ with homogenous Dirichlet boundary condition. The optimality system from section 2 is given by

$$
\left\{\begin{array}{l}
-\triangle \vec{y}^{*}=\vec{u}^{*},\left.\quad \vec{y}^{*}\right|_{\partial \Omega}=\overrightarrow{0}, \\
-\triangle \vec{p}^{*}=\vec{y}_{d}-\vec{y}^{*},\left.\quad \vec{p}^{*}\right|_{\partial \Omega}=\overrightarrow{0}, \\
\vec{p}^{*}=\beta^{*} \vec{u}^{*}, \quad \beta^{*}=\max \left(\alpha,\left|\vec{p}^{*}\right|\right) .
\end{array}\right.
$$


TABLE 1. super-linear convergence

\begin{tabular}{|c|c|c|c|c|c|}
\hline iter. number & 1 & 2 & 3 & 4 & 5 \\
\hline$\left\|y^{k}-y^{*}\right\|$ & 1.982 & $4.195 \mathrm{e}-1$ & $5.183 \mathrm{e}-2$ & $5.371 \mathrm{e}-3$ & $2.812 \mathrm{e}-7$ \\
\hline$\frac{\left\|y^{k}-y^{*}\right\|}{\left\|y^{k-1}-y^{*}\right\|}$ & & $2.117 \mathrm{e}-1$ & $1.236 \mathrm{e}-1$ & $1.0367 \mathrm{e}-1$ & $5.235 \mathrm{e}-5$ \\
\hline$\left\|p^{k}-p^{*}\right\|$ & 3.575 & $3.877 \mathrm{e}-1$ & $2.534 \mathrm{e}-2$ & $3.437 \mathrm{e}-3$ & $4.974 \mathrm{e}-8$ \\
\hline$\frac{\left\|p^{k}-p^{*}\right\|}{\left\|p^{k-1}-p^{*}\right\|}$ & & $1.084 \mathrm{e}-1$ & $6.537 \mathrm{e}-2$ & $1.356 \mathrm{e}-1$ & $1.437 \mathrm{e}-5$ \\
\hline$\left\|\beta^{k}-\beta^{*}\right\|$ & $1.982 \mathrm{e}-1$ & $3.094 \mathrm{e}-1$ & $1.626 \mathrm{e}-1$ & $1.718 \mathrm{e}-3$ & $4.236 \mathrm{e}-7$ \\
\hline$\frac{\left\|\beta^{k}-\beta^{*}\right\|}{\left\|\beta^{k-1}-\beta^{*}\right\|}$ & & 1.561 & $5.255 \mathrm{e}-1$ & $1.057 \mathrm{e}-2$ & $2.466 \mathrm{e}-4$ \\
\hline
\end{tabular}

TABLE 2. number of iterations for Algorithm 1

\begin{tabular}{|r|r|r|r|r|}
\hline & $\alpha=0.1$ & 0.01 & 0.001 & 0.0001 \\
\hline$p^{0}=0.1 \alpha$ rand & 5 & 6 & 6 & 7 \\
\hline$p^{0}=1 \alpha$ rand & 5 & 6 & 6 & 8 \\
\hline$p^{0}=10 \alpha$ rand & 5 & 7 & 8 & 26 \\
\hline$p^{0}=100 \alpha$ rand & 6 & 7 & 9 & 37 \\
\hline$p^{0}=1000 \alpha$ rand & 6 & 8 & 12 & NA \\
\hline
\end{tabular}

Our first example involves the construction of an the exact solution as follows: given a function $\vec{p}^{*}$ with enough regularity, define $\beta^{*}=\max \left(\alpha,\left|\vec{p}^{*}\right|\right), \vec{u}^{*}=\frac{1}{\beta^{*}} \vec{p}^{*}$, $\vec{y}^{*}=(-\triangle)^{-1} \vec{u}^{*}$ and let $\vec{y}_{d}=\vec{y}^{*}-\triangle \vec{p}^{*}$. Then for any given initial guess $\vec{p}^{0}, \beta^{0}$, we can apply Algorithm 1 to find the solution to this problem, and we choose the residual level as a stop criterion.

In the numerical test, we choose $\vec{p}^{*}=\alpha(\sin (4 \pi x y), \sin (8 \pi x y)+x(1-x) y(1-y))$, and compute $\beta^{*}, \vec{u}^{*}, \vec{y}^{*}$ and $\vec{y}_{d}$ accordingly. For this example, we observe superlinear convergence in Table 1 for $\alpha=0.001$ and random initial guess $\vec{p}^{0}, \beta^{0}$. It is also noted from Table 2, that for a decreasing sequence of $\alpha$ values and large random initial guesses, the algorithm always convergence in a few steps except in the most extreme case.

Our second example is slightly different. This time we do not construct an exact solution to compare with our approximation. Instead we let $\vec{y}_{d}=c \alpha(\sin (\pi x y)+$ $x+3 y, \sin (2 \pi x)+\cos (2 \pi y))$, where $c$ is a constant between 10 to 100 to make the constraint active at the optimal solution. We observe that for a relatively large $\alpha$ 
TABLE 3. number of iterations for Algorithm 2

\begin{tabular}{|r|r|r|r|r|}
\hline$\alpha^{k}$ & 0.1 & 0.01 & 0.001 & 0.0001 \\
\hline$\alpha=0.001$ & 3 & 3 & 10 & \\
\hline$\alpha=0.0001$ & 3 & 3 & 4 & 9 \\
\hline
\end{tabular}

$(\alpha>0.02)$, the convergence is not sensitive to the initial guess. After decreasing $\alpha$, the convergence region becomes smaller. With randomly chosen initial data the algorithm may not converge. We therefore introduce a continuation technique which is explained in Algorithm 2. With this procedure the algorithm is robust. We use it with $\alpha^{0}=0.1$ and $\rho=0.1$ for the following two test problems:

(1) $\alpha=0.001, c=30$, i.e., $\vec{y}_{d}=0.03(\sin (\pi x y)+x+3 y, \sin (2 \pi x)+\cos (2 \pi y))$.

(2) $\alpha=0.0001, c=80$, i.e., $\vec{y}_{d}=0.008(\sin (\pi x y)+x+3 y, \sin (2 \pi x)+\cos (2 \pi y))$.

In Table 3, we record the number of iterations at each sub-problem as $\alpha^{k}$ becomes small with $\left(\vec{p}^{k}, \beta^{k}\right)$ as the initial guess. The total number of iterations is less than 20. Figure 1 depicts the Lagrange multiplier for each of the two cases (we plot $\beta=\alpha+\lambda)$. As expected these quantities are $W^{1, p}$ regular.

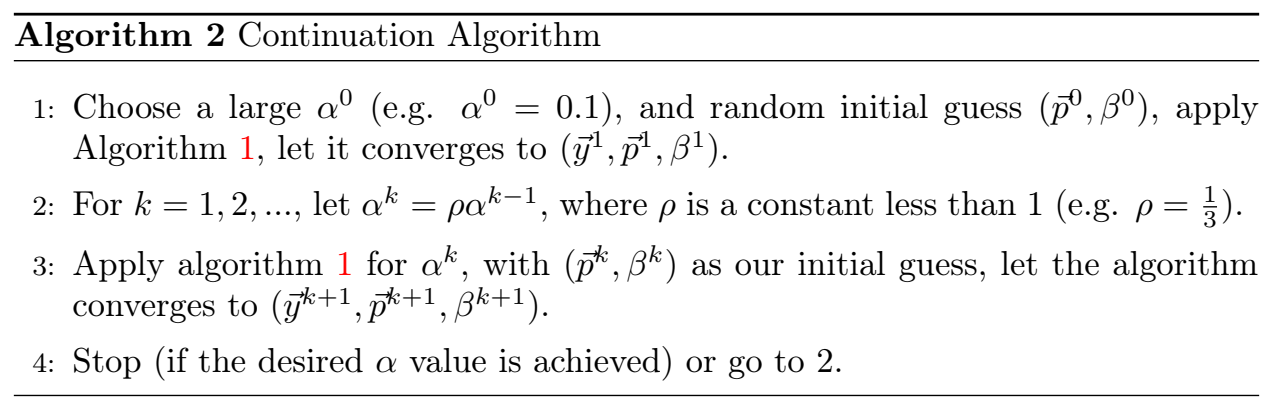

FIGURE 1. Lagrange multiplier in second example
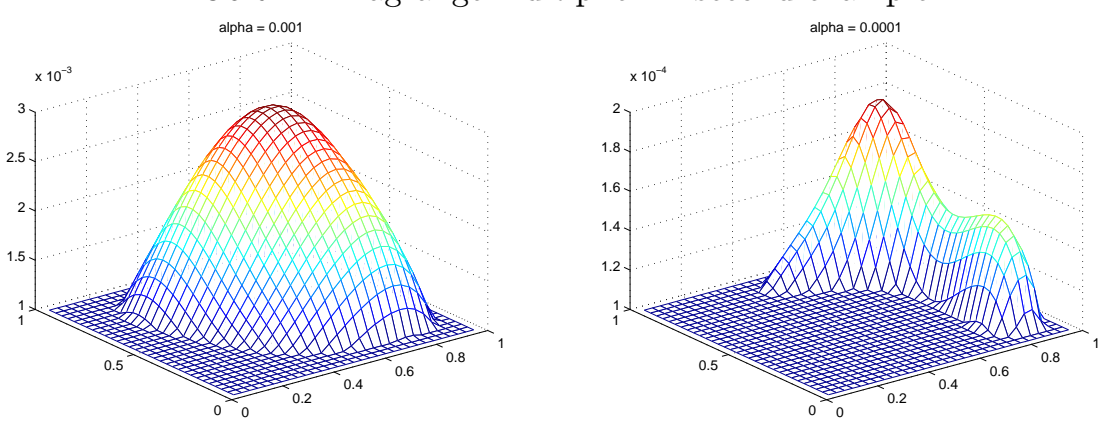

For the last numerical example we consider the Lamé system from the theory of linearly elastic materials. The governing equation is given by

$$
-\nu \triangle \vec{y}-(\lambda+\nu) \nabla \operatorname{div} \vec{y}=\vec{u},\left.\quad \vec{y}\right|_{\partial \Omega}=\overrightarrow{0},
$$


TABLE 4. super-linear convergence for the linear elasticity system

\begin{tabular}{|r|r|r|r|r|r|}
\hline iter. number & 6 & 7 & 8 & 9 & 10 \\
\hline$\left\|y^{k}-y^{k-1}\right\|$ & $7.726 \mathrm{e}-5$ & $1.107 \mathrm{e}-6$ & $1.393 \mathrm{e}-8$ & $3.749 \mathrm{e}-14$ & $2.349 \mathrm{e}-20$ \\
\hline$\frac{\left\|y^{k}-y^{k-1}\right\|}{\left\|y^{k-1}-y^{k-2}\right\|}$ & & $1.432 \mathrm{e}-1$ & $1.285 \mathrm{e}-2$ & $2.692 \mathrm{e}-6$ & $6.266 \mathrm{e}-7$ \\
\hline$\left\|p^{k}-p^{k-1}\right\|$ & $2.636 \mathrm{e}-5$ & $2.912 \mathrm{e}-7$ & $5.148 \mathrm{e}-10$ & $1.517 \mathrm{e}-15$ & $1.475 \mathrm{e}-20$ \\
\hline$\frac{\left\|p^{k}-p^{k-1}\right\|}{\left\|p^{k-1}-p^{k-2}\right\|}$ & & $1.105 \mathrm{e}-2$ & $1.768 \mathrm{e}-3$ & $2.947 \mathrm{e}-6$ & $9.723 \mathrm{e}-6$ \\
\hline$\left\|\beta^{k}-\beta^{k-1}\right\|$ & $1.844 \mathrm{e}-5$ & $2.613 \mathrm{e}-7$ & $3.628 \mathrm{e}-9$ & $1.282 \mathrm{e}-13$ & $9.158 \mathrm{e}-21$ \\
\hline $\begin{array}{r}\left\|\beta^{k}-\beta^{k-1}\right\| \\
\left\|\beta^{k-1}-\beta^{k-2}\right\|\end{array}$ & & $1.417 \mathrm{e}-2$ & $1.388 \mathrm{e}-2$ & $3.524 \mathrm{e}-5$ & $7.144 \mathrm{e}-8$ \\
\hline
\end{tabular}

where $\nu$ and $\lambda$ are the Lamé coefficients. Let the controls be constrained to satisfy $|\vec{u}(\mathbf{x})| \leq 1$, a.e. $\mathbf{x} \in \Omega$. The optimality system reads

$$
\begin{cases}-\nu \triangle \vec{y}-(\lambda+\nu) \nabla \operatorname{div} \vec{y}=\vec{u}, & \left.\vec{y}\right|_{\partial \Omega}=\overrightarrow{0}, \\ -\nu \triangle \vec{p}-(\lambda+\nu) \nabla \operatorname{div} \vec{p}=\vec{y}_{d}-\vec{y}, & \left.\vec{p}\right|_{\partial \Omega}=\overrightarrow{0}, \\ \vec{p}=\beta \vec{u}, \beta=\max (\alpha,|\vec{p}|) . & \end{cases}
$$

We apply Algorithm 1 to solve the above optimality system. In our numerical test, the Lamé coefficients are chosen to be $\nu=1, \lambda=1000, \alpha=0.01, \vec{y}_{d}=$ $\left(5+\sin (x y), 2-\cos \left(2 x-y^{2}\right)\right)$. The initial guess is taken to be $\beta^{0}=\alpha, \vec{p}^{0}=\overrightarrow{0}$. To discretize the optimality system, we use conforming $P_{2}$ finite element to avoid possible locking phenomena due to $\lambda \gg \nu$. From Table 4, we can observe that the number of iteration is larger than for the first two numerical examples, but the algorithm again converges super-linearly at the end.

\section{ACKNOWLEDGMENT}

The authors would like to thank the reviewers for their constructive feedback and for suggesting the projection argument that is now used to prove the optimality conditions.

\section{REFERENCES}

[1] R. Adams, J. Fournier, Sobolev Spaces, Academic Press, 2003.

[2] J. E. Dennis and R.B. Schnabel, Numerical Methods for Unconstrained Optimization and Nonlinear Equations, Prentice-Hall, Englewood Cliffs, 1983.

[3] D. Gilbarg and N. Trudinger, Elliptic Partial Differential Equations of Second Order, Springer Verlag, Berlin, 1983.

[4] M. Hintermüller and M. Hinze, A SQP-semismooth Newton-type algorithm applied to control of the instationary Navier-Stokes system subject to control constraints, SIAM J. Optim. 16 (2006), pp. 1177-1200.

[5] M. Hintermüller, K. Ito, and K. Kunisch, The Primal-Dual Active Set Strategy as a semismooth Newton Method, SIAM J. Optim., 13 (2003), 865-888. 
[6] M. Hinze, A variational discretization concept in control constrained optimization: the linearquadratic case, Comput. Optim. Appl. 30 (2005), pp. 45-61.

[7] K. Ito, and K. Kunisch, The primal-dual active set method for nonlinear optimal control problems with bilateral constraints, SIAM J. Control Optim. 43 (2004), pp. 357-376.

[8] K. Ito, and K. Kunisch, On the Lagrange Multiplier Approach to Variational Problems and Applications, SIAM, Philadelphia, 2008.

[9] K. Kunisch and X. Lu, Optimal Control on an Elliptic System with Convex Polygonal Control Constraints, acceptted by IMA Numer. Anal.

[10] E. Lieb and M. Loss, Analysis, volume 14 of Graduate Studies in Mathematics. American Mathematical Society, Providence, RI, second edition, 2001.

[11] J. C. De Los Reyes and K. Kunisch, Optimal control of partial differential equations with affine control constraints, Control and Cybernetic, 38 (2009), pp. 1217-1249.

[12] A. Rösch, Error estimates for parabolic optimal control problems with control constraints, Z. Anal. Anwendungen 23 (2004), pp. 353-376.

[13] W. Rudin, Principles of Mathematical Analysis, McGraw-Hill, 1976.

[14] M. Ulbrich, Constrained optimal control of Navier-Stokes flow by semismooth Newton methods, Systems and Control Letters, 48, (2003), pp.297-311.

[15] M. Ulbrich, Semismooth Newton methods for operator equations in function spaces, SIAM J. Optim., 13 (2003), 805-842.

[16] M. Ulbrich, Semismooth Newton Methods for Variational Inequalities and Constrained Optimization Problems in Function Spaces, SIAM, Philadelphia, 2011.

[17] S. Ulbrich, Global convergence of trust-region interior-point algorithms for infinitedimensional nonconvex minimization subject to pointwise bounds, SIAM J. Control Optim., 37(1999), 731-764.

[18] B. Vexler and W. Wollner, Adaptive finite elements for elliptic optimization problems with control constraints, SIAM J. Control Optim. 47 (2008), pp. 509-534.

[19] D. Wachsmuth, Sufficient second-order optimality conditions for convex control constraints, J. Math. Anal. Appl. 319 (2006), pp. 228-247.

[20] D.Wachsmuth, Numerical solution of optimal control problems with convex control constraints. In: Systems, Control, Modeling and Optimization, eds.: Ceragioli, Dontchev, Furuta, Marti, Pandolfi, pp. 319-327. Springer (2006).

[21] D. Wachsmuth, Optimal control problems with convex constraints. In: Control of Coupled Partial Differential Equations, ISNM Vol. 155, eds.: K. Kunisch, G. Leugering, J. Sprekels, F. Tröltzsch, pp. 311-328, Birkhäuser (2007).

Institute of Mathematics and Scientific Computing, University of Graz, HeinrichStrasse 36 A-8010 Graz, Austria

E-mail address: karl.kunisch@uni-graz.at

Corresponding author. School of Mathematics and Statistics, Wuhan University, Wuhan 430072, P.R. China.

E-mail address: xllv.math@whu.edu.cn 